\title{
Plasma deposition of Ultrathin polymer films on carbon nanotubes
}

\author{
Donglu Shi ${ }^{\mathrm{a})}$ \\ Department of Materials Science and Engineering, University of Cincinnati, Cincinnati, Ohio 45221 \\ Jie Lian \\ Department of Nuclear Engineering and Radiological Science, University of Michigan, Ann Arbor, \\ Michigan 48109 \\ Peng He \\ Department of Materials Science and Engineering, University of Cincinnati, Cincinnati, Ohio 45221 \\ L. M. Wang \\ Department of Nuclear Engineering and Radiological Science, University of Michigan, Ann Arbor, \\ Michigan 48109 \\ Wim J. van Ooij \\ Department of Materials Science and Engineering, University of Cincinnati, Cincinnati, Ohio 45221 \\ Mark Schulz and Yijun Liu \\ Department of Mechanical Engineering, University of Cincinnati, Cincinnati, Ohio 45221 \\ David B. Mast \\ Department of Physics, University of Cincinnati, Cincinnati, Ohio 45221
}

(Received 21 May 2002; accepted 18 October 2002)

\begin{abstract}
Ultrathin films of pyrrole were deposited on the surfaces of carbon nanotubes using a plasma polymerization treatment. High-resolution electron transmission microscopy images revealed that an extremely thin film of the polymer layer $(2 \sim 7 \mathrm{~nm})$ was uniformly deposited on the outer and inner surfaces of the nanotubes. The nanotubes of all sizes exhibited equally uniform ultrathin films, indicating well-dispersed nanotubes in the fluidized bed reactor during the plasma treatment. In particular, the inner wall of the nanotube was also coated with a uniform ultrathin film of only $\sim 1-3$ $\mathrm{nm}$. Time-of-flight secondary ion mass spectroscopy experiments confirmed the highly branched and cross-linked polymer thin films on the carbon nanotubes. The plasma deposition mechanism is discussed in this letter. ㅇ 2002 American Institute of Physics. [DOI: 10.1063/1.1527702]
\end{abstract}

The development of surface nanostructures will be one of the key engines that drive our technological society in the 21 st century. This rapidly growing area focuses on tailoring a nanoparticle surface structure for specific and unique properties. ${ }^{1-6}$ One of the important developments in nanostructures is the synthesis of carbon nanotubes. Carbon nanotubes are intrinsically superelastic one-dimensional structures that are chemically inert, and they possess electrochemical, piezoelectric, tunable electronic, and high thermal conductivity properties. ${ }^{7}$ These properties make carbon nanotubes the potential successor to carbon fibers in composites, silicon in electronic devices, ionic polymers for actuators ${ }^{8}$ and a myriad of other technologies for sensors. Unfortunately, the surface of the nanotube is often not ideal for a particular application. The ability to deposit wellcontrolled coatings on nanotubes would offer a wide range of technological opportunities, based on changes to both the physical and chemical properties of the nanotubes and nanoparticles.

The coating nanotechnology being reported in this letter is also critically important for active materials development and in the design of smart nanocomposites. Nanocomposite film actuators are being investigated by coating carbon nanotube ropes with a structural polymer electrolyte to improve ion exchange and transduction efficiency. ${ }^{9,10}$ Coatings may

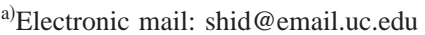

also be able to increase the piezoelectric properties of nanotubes ${ }^{11}$ for use in high-frequency strain sensing applications. An innovative plasma polymerization coating process $^{12}$ that can be used for many of the above applications is discussed subsequently.

Generally, nanoparticles are difficult to handle in the plasma polymerization coating process due to aggregation and the large surface area per unit mass of the powder. ${ }^{13}$ However, a fluidized bed reactor is an ideal tool for gasparticle reactions due to the intensive mass and heat transfer between the two phases, short reaction time, omnidirectional deposition, and flat temperature profile. ${ }^{14}$ Therefore, the combination of plasma polymerization and the fluidized bed process represents an innovative approach for low temperature surface modification of nanoparticles. 15,16

In this experiment, we used Pyrograf III PR-24-PS and PR-24-HT ${ }^{17}$ nanotubes as substrates. These nanotubes have the same structure and purchased from Pyrograf Products, Inc. The plasma reactor for thin film deposition of nanotubes has been introduced previously. ${ }^{12}$ The vacuum chamber of the plasma reactor consists of a Pyrex ${ }^{\mathrm{TM}}$ glass column about $80 \mathrm{~cm}$ in height and $6 \mathrm{~cm}$ in internal diameter. The carbon nanotubes are vigorously stirred at the bottom of the tube and thus the surfaces of nanotubes can be continuously rotated and exposed to the plasma for thin film deposition during the plasma polymerization process. A magnetic bar was used to stir the powders. The gases and monomers were in- 


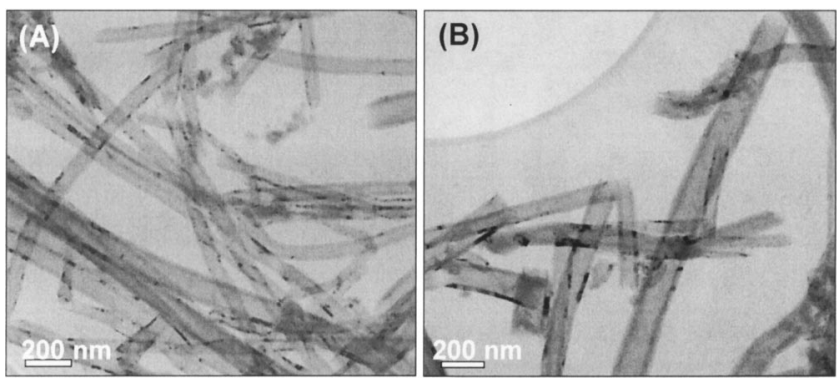

FIG. 1. Bright-field TEM images of the original uncoated carbon nanotubes of Pyrograf III PR-24-PS PR-24-HT (a) and Pyrograf III PR-24-PS (b).

troduced from the gas inlet during the plasma cleaning treatment or plasma polymerization. The system pressure was measured by a thermocouple pressure gauge. A rf power generator operating at $13.56 \mathrm{MHz}$ was used for the plasma film deposition.

Before the plasma treatment, the chamber pressure was pumped down to less than $5 \mathrm{~Pa}$, at which time the monomer vapors were introduced into the reactor chamber. The operating pressure was adjusted by the mass flow controller. Pyrrole was used as the monomer for plasma polymerization. To be able to distinguish the deposited polymer thin film and the surface of carbon nanotubes, we introduced a small fraction of $\mathrm{C}_{6} \mathrm{~F}_{14}$ to copolymerize with the pyrrole monomer. In this way, we will be able to characterize the deposited thin film in the time-of flight secondary ion mass spectrometry (TOFSIMS) experiments. During the plasma polymerization process, the rf power was $15 \mathrm{~W}$ and the system pressure was 30 $\mathrm{Pa}$. The plasma treatment time was 30 min per batch of $0.3 \mathrm{~g}$ of powder.

After the plasma treatment, the carbon nanotubes were examined using transmission electron microscopy (TEM) and TOFSIMS. The high-resolution TEM (HRTEM) experiments were performed using a JEOL JEM 2010F electron microscope with a field emission source. The accelerating voltage was $200 \mathrm{kV}$. The nanotubes were dispersed in methanol and suspended on a perforated carbon film supported by $\mathrm{Cu}$ grids. Bright-field and high-resolution imaging techniques were used to characterize the features of both the original and the coated carbon nanotubes. TOFSIMS was performed on a Ion-Tof model IV equipped with a $25 \mathrm{keV}$ $69 \mathrm{Ga}^{+}$source. The mass resolution of the instrument was 8000 at 28 amu. Positive and negative spectra were collected in the mass range of 0 to $1000 \mathrm{amu}$. The spectra were acquired by rastering the beam over an areas of 300 $\times 300 \mu \mathrm{m}$ of bundle of the untreated or treated carbon nanotubes.

Figure 1 shows the bright-field TEM images of the original uncoated Pyrograf III PR-24-HT [Fig. 1(a)] nanotubes and the Pyrograf III PR-24-PS carbon nanotubes [Fig. 1(b)]. As can be seen in this figure, both Pyrograf III carbon nanotubes have similar sized features with a hollow channel. The Pyrograf III PR-24-HT nanotubes have slightly smaller outside diameters averaging about $70 \mathrm{~nm}$, and they are quite uniformly distributed. The Pyrograf III PR-24-PS carbon nanotubes have outside diameters ranging between 40 and $120 \mathrm{~nm}$. Some nanotubes become curved during their growth with the open ends. An HRTEM image [Fig. 2(a)] of the original Pyrograf III PR-24-HT carbon nanotubes shows the graphite structure with the interlayer spacing $d_{002}=0.34 \mathrm{~nm}$.

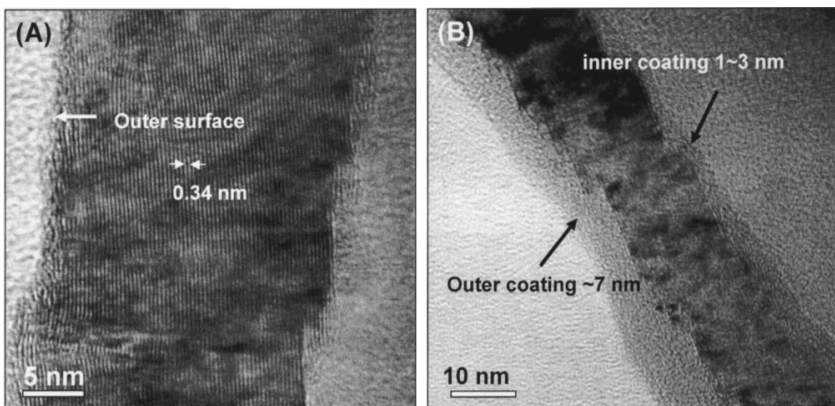

FIG. 2. HRTEM images of Pyrograf III PR-24-PS PR-24-HT nanotube. (a) The fragments of the wall with inclined planes (002) showing lattice space on the outer and inner surfaces of uncoated Pyrograf III PR-24-PS PR24-HT nanotubes with slight roughness $(<1 \mathrm{~nm})$ on the surface. (b) An ultrathin film of pyrrole can be observed on both outer and inner surfaces of coated Pyrograf III PR-24-PS PR-24-HT nanotubes.

Based on the bright-field TEM and HRTEM images, the wall thickness of the nanotubes can be estimated to be about 20 $\sim 30 \mathrm{~nm}$ for both the Pyrograf III PR-24-HT and Pyrograf III PR-24-PS carbon nanotubes.

Nanotubes with axially parallel graphite layers (not shown here) and nanotubes with axially parallel graphite layers oriented at an angle to the tube axis [Fig. 2(a)] were observed. The edge dislocations can be seen due to the disorder of the graphite layers (002). It is noticed that both the outer and inner surfaces terminate at the graphite (002) layer without the addition of a surface layer, for the originally uncoated nanotubes [Fig. 2(a)]. The bright-field and HRTEM images of these nanotubes after plasma treatment are shown in Fig. 2(b) (Pyrograf III PR-24-PS PR-24-HT nanotubes) and Fig. 3 (Pyrograf III PR-24-PS carbon nanotubes), respectively. An ultrathin film amorphous layer can be clearly seen covering both the inner and outer surfaces of the Pyrograf III PR-24-HT nanotubes [Fig. 2(b)], but only covering the outer surface of the Pyrograf III PR-24-PS carbon nanotubes [Fig. 3(a)]. The thin film is uniform on both surfaces, with a larger thickness on the outer wall $(7 \mathrm{~nm})$ than on the inner wall $(1 \sim 3 \mathrm{~nm})$ surface [Fig. 2(b)]. The thickness of ultrathin film is approximately $2 \sim 7 \mathrm{~nm}$ surrounding the entire nanotube surface of both the Pyrograf III PR-24-HT and Pyrograf III PR-24-PS carbon nanotubes. The film is also thicker and more uniform than the roughness $(<1 \mathrm{~nm})$ on the outer surface of the carbon nanotubes [Fig. 2(a)]. In Fig. 3(b), we show the HRTEM image of a coated Pyrograf III PR-24-PS carbon nanotube. The lattice image of graphite can
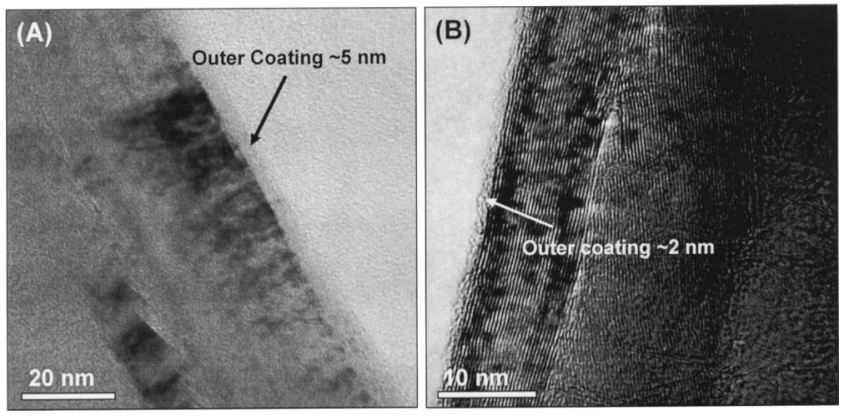

FIG. 3. The bright-field TEM (a) and HRTEM images (b) showing the coating on the outer surface of the Pyrograf III PR-24-PS carbon nanotube. The lattice image of carbon can be clearly seen with an extremely thin layer of polymer film $(\sim 2 \mathrm{~nm})$ on the outer surface of the coated Pyrograf III PR-24-PS carbon nanotubes. 

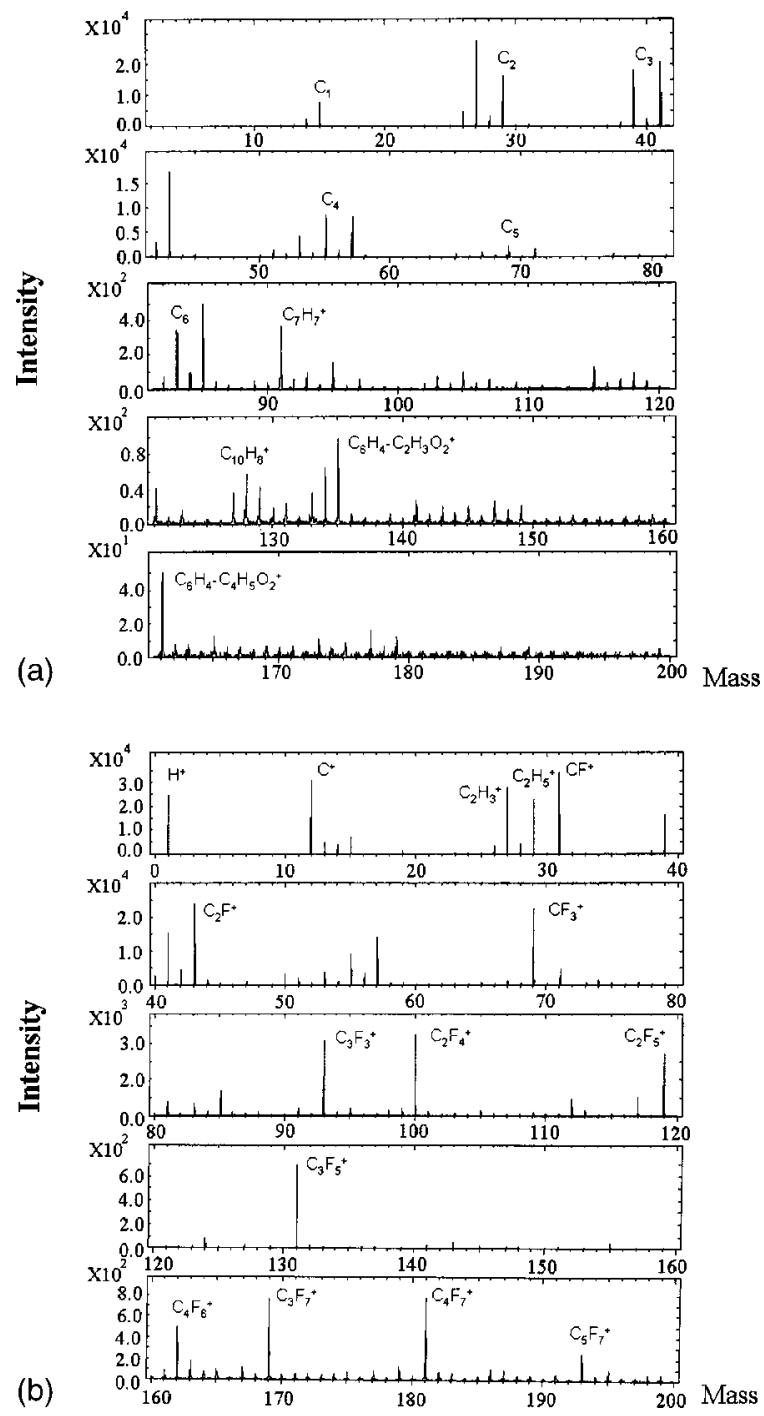

FIG. 4. TOFSIMS spectra of (a) untreated and (b) treated carbon nanotubes.

be clearly seen with an extremely thin layer of polymer film on its surface.

To confirm the TEM observations shown in Fig. 1-Fig. 3, TOFSIMS was carried out to study the surface films of the nanotubes. Figure 4(a) shows part of the TOFSIMS spectra of untreated Pyrograf III PR-24-HT carbon nanotubes. The spectrum of the untreated nanotubes show an appreciable intensity of carbon, hydrogen, and oxygen, which is a characteristic of a untreated natural surface. In Fig. 4(b) one can see that the treated nanotubes have strong carbon-fluorine peaks, indicating the surface coating of the nanotubes, and consistent with the HRTEM data presented in Fig. 1-Fig. 3. In particular, the spectrum in Fig. 4(b) shows carbonfluorine in the forms of $\mathrm{C}_{4} \mathrm{~F}_{7}^{+}, \mathrm{C}_{3} \mathrm{~F}_{7}^{+}, \mathrm{C}_{4} \mathrm{~F}_{6}^{+}$, and $\mathrm{C}_{5} \mathrm{~F}_{7}^{+}$, indicating highly branched and cross-linked polymer structure in the deposited thin film. It is to be noted that the fluorine shown in the TOFSIMS spectrum can only be part of the monomer introduced during the plasma coating process, a strong indication of polymerized film on the carbon nanotubes.

One of the critical issues addressed in this study is the deposition of thin films on the inner tube surface. In our previous studies, we have successfully deposited ultra thin films on the nanoparticle surfaces of alumina and $\mathrm{ZnO}^{12}$
However, in this work, the deposition must take place in the inner tube whose diameter is only about $20 \mathrm{~nm}$. The length of these tubes is on the order of several microns. In order to obtain a uniform coating at the inner wall surfaces, the fluidization of the nanotubes and the plasma condition must be critically controlled. In our plasma coating process, both energy terms (surface energy of the nanotube and the surface tension of the polymer) were balanced by controlling the plasma coating parameters, including electron density, temperature, and energy density. The gas pressure must be moderate for a low collision rate on the nanotube inner and outer surfaces. As shown in Fig. 2(b), there is an extremely thin $(1 \sim 3 \mathrm{~nm})$ polymer film deposited on the inner wall tube surface while a relatively thicker film is deposited on the outer surface. This is an indication of the deposition rate difference within and outside the nanotube. Because of the nanoscale diameter of the tube, for a given gas pressure, the collision frequency must be reduced inside the nanotube, resulting in a lower deposition rate. However, this deposition rate difference does not appear to be great for both inner and outer surfaces, as evidenced in Fig. 2(b).

In summary, we have deposited an ultrathin polymer film on the inner and outer surfaces of carbon nanotubes by means of a plasma polymerization treatment. The polymer layer is not only uniform on both inner and outer surfaces, but it is also deposited in an extremely thin layer of 2 $\sim 7 \mathrm{~nm}$. TOFSIMS spectra confirmed the polymer nature of the deposited thin films. By controlling the plasma coating conditions, the deposition rate can be closely controlled so that the film thickness on both the inner and outer wall surfaces is uniform and nearly identical.

The TEM analyses were conducted at the Electron Microbeam Analysis Laboratory at the University of Michigan, Ann Arbor, Michigan. This research was supported in part by a grant from NSF, DMII division, No. DMI-9713715.

${ }^{1}$ R. W. Siegel, Nanostruct. Mater. 3, 1 (1993).

${ }^{2}$ G. C. Hadjipanayis and R. W. Siegel, Nanophase materials, Synthesisproperties-applications (Kluwer, Dordrecht, 1994).

${ }^{3}$ G. M. Whitesides, J. P. Mathias, and C. T. Seto, Science 254, 1312 (1991).

${ }^{4}$ C. D. Stucky and J. E. MacDougall, Science 247, 669 (1990).

${ }^{5}$ H. Gleiter, Nanostruct. Mater. 6, 3 (1995).

${ }^{6}$ Nanotechnology, edited by A. T. Wolde (STT Netherlands Study Center for Technology Trends, The Hague, The Netherlands, 1998).

${ }^{7}$ G. Timp, Nanotechnology (AIP, Springer, Melville, New York, 1998).

${ }^{8}$ Y. Bar-Cohen, 42nd AIAA Structures, Dynamics, and Materials Conference (SDM), Gossamer Spacecraft Forum (GSF), Seattle, WA, 2001, p. $1-10$.

${ }^{9}$ D. D. Mazzoldi and R. H. Baughman, Proc. SPIE , 25 (2000).

${ }^{10}$ J. Fraysse, A. I. Minett, G. Gu, S. Roth, et al., "Towards the demonstration of actuator properties of a single carbon nanotube," Current Applied Physics, 1, 407 (2001).

${ }^{11}$ N. G. Lebedev, I. V. Zaporotskova, and L. A. Chernozatonskii, On the Estimation of Piezoelectric Modules of Carbon and Boron Nitride Nanotubes, Volograd State University, 400062 Volograd, Russia, and Institute of Biochemical Physics of RAS, 117334, Moscow, Russia, 2001.

${ }^{12}$ D. Shi and W. J. v. Ooij, Appl. Phys. Lett. 78, 1243 (2001).

${ }^{13}$ N. Inagaki, S. Tasaka, and K. Ishii, J. Appl. Polym. Sci. 48, 1433 (1993).

${ }^{14}$ C. Bayer, M. Karches, A. Mattews and P. R. Von Rohr, Chem. Eng. Technol. 21, 427 (1998).

${ }^{15}$ S. Eufinger, W. J. van Ooij, and T. H. Ridgway, J. Appl. Polym. Sci. 61, 1503 (1996).

${ }^{16}$ W. J. van Ooij, S. Eufinger, and T. H. Ridgway, Plasma and Polymers 1, 231 (1996).

${ }^{17}$ Applied Sciences, Inc., 141 W. Xena Ave., P.O. Box 579, Cedarville, OH 45314-0579. 